\title{
Distribution of the Lingual Tonsils of Cattle Designated as Specified Risk Materials
}

\author{
Keiko $\mathrm{KATO}^{1)}$ and Yasushi SAWADA ${ }^{2)}$ \\ 1) Animal Care and Consultation Center, Bureau of Social Welfare and Public Health, Tokyo Metropolitan Government, 2-9-11, \\ Hachimanyama, Setagaya-ku,Tokyo 156-0056 and ${ }^{2}$ Shibaura Meat Sanitary Inspection Station, Bureau of Social Welfare and Public \\ Health, Tokyo Metropolitan Government, 2-7-19 Kounan, Minato-ku, Tokyo 108-0075, Japan
}

(Received 29 August 2007/Accepted 21 November 2007)

ABSTRACT. The tonsils of cattle, including palatine tonsils, pharyngeal tonsils, tubal tonsils and lingual tonsils, are designated as specified risk materials (SRM). However, the detailed distribution of lingual tonsils in cattle is unknown. We therefore histologically examined their distribution in 198 tongue specimens from cattle. The examinations confirmed that the presence of lingual tonsils was limited to the tissue of the lamina propria on the dorsal and lateral aspects of the tongue, not reaching the muscular layer below. More than $90 \%$ of the lingual tonsils were located between the distribution center of the vallate papillae and the radix linguae (root of the tongue). However, they were also found in the area extending from the lingual torus to the rostral-most vallate papilla in an individual, suggesting that the complete removal of the lingual tonsils requires elimination of the lamina propria extending from the lingual torus to the radix linguae.

KEY WORDS: BSE, cattle, lingual tonsils, specified risk materials (SRM), tongue.

J. Vet. Med. Sci. 70(3): 251-254, 2008

Bovine spongiform encephalopathy (BSE), a type of transmissible spongiform encephalopathy (TSE), was reported first in the U.K. in 1987 [10]. By the end of 2006, more than 180,000 cases had been reported in United Kingdom. Then, the first case of a variant Creutzfeldt-Jakob diease (vCJD) caused by BSE was reported in $1996[1,12]$. In response to the identification in 2001 of BSE in nativeborn cattle, active surveillance on all slaughtered cattle started in October of that year in Japan. Up to June 30, 2007, 33 BSE-confirmed cases including the first BSE case were found in Japan.

Eliminating SRM from cattle carcasses is an important measure for protecting human and livestock health from the risk of BSE. In the European Union, the tonsils have been designated as SRM and removed from beef carcasses since 2000 [2]. Also in Japan, the head region with the tonsils have been eliminated in accordance with the Law on Special Measures Against BSE since 2002 [6].

In 2002, the British Food Standards Agency (FSA), using an assay several hundred times more sensitive than the standard assay, found a low level of BSE infectivity in cattle tonsils [7]. As a result, in November of that year, the Ministry of Health, Labour and Welfare of Japan issued a warning not to touch the tonsils while removing the tongue from the head region. Although additional notice to eliminate the lingual tonsils adequately was issued in 2005 , this did not involve the descriptions of the concrete existence position in the lingual tonsils.

There are four types of tonsils - palatine tonsils, pharyngeal tonsils, lingual tonsils and tubal tonsils [4]. The palatine and pharyngeal tonsils are visible to the unaided

\footnotetext{
* Correspondence to: Sawada, Y., Shibaura Meat Sanitary Inspection Station, Bureau of Social Welfare and Public Health, Tokyo Metropolitan Government, 2-7-19, Kounan, Minato-ku, Tokyo 108-0075, Japan.

e-mail:Yasushi_Sawada@member.metro.tokyo.jp
}

eye. The pharyngeal and tubal tonsils are distributed in the tissue of the rhinopharynx and are therefore removed as a part of the head region. However, the lingual tonsils, which exist in the tongue as histologically independent lymph nodules, cannot be identified macroscopically [8]. Slaughter practices in the U.S. follow the guideline that there are no lingual tonsils rostral to the vallate papilla closest to the radix linguae. Accordingly, during slaughter the tongue is severed at the most caudal vallate papilla and the rostral part is deemed safe for consumption [9]. However, it has been reported that lingual tonsilar material has been found rostral to the most caudal vallate papilla [5]. So far, a detailed description of lingual tonsillar distribution has only been given by Wells et al.

Here, we report on our histological examination of the distribution of lingual tonsils in cattle and discuss their removal methods.

\section{MATERIALS AND METHODS}

The specimens examined were tongues from 198 cattle removed after slaughter inspection that included a BSE test. The breakdown of samples by age, breed and sex is shown in Table 1. The specimens were divided by age into the following four categories: (1) $\mathrm{x}<12$ months old, (2) 12 months $\leq \mathrm{x}<20$ months, (3) 20 months $\leq \mathrm{x}<30$ months and (4) 30 months $\leq \mathrm{x}$. In addition, 15 tongues that had already had the surface mucosa removed for marketing (so-called "peeled tongues") were checked for the persistence of lingual tonsils.

Tongues were refrigerated for $18 \mathrm{hr}$ after slaughter. The tissue examined consisted of the area extending from the tip of the lingual torus to the radix linguae on the left or right lateral aspect, as divided by the dorsal midline. Tongues were cut into 5-mm-wide strips from the center of the most 
Table 1. Breakdown of specimens examined

\begin{tabular}{|c|c|c|c|c|c|c|c|c|c|c|}
\hline \multirow{2}{*}{$\begin{array}{l}\text { Age in } \\
\text { months }\end{array}$} & \multicolumn{3}{|c|}{ Japanese beef cattle } & \multicolumn{3}{|c|}{ Crossbred cattle } & \multicolumn{3}{|c|}{ Dairy cattle } & \multirow[b]{2}{*}{ Total } \\
\hline & Bull & Steer & Cow & Bull & Steer & Cow & Bull & Steer & Cow & \\
\hline$<12$ & & 1 & 1 & & & 1 & 6 & 17 & & 26 \\
\hline$<20$ & & & & & 1 & & & 28 & & 29 \\
\hline$<30$ & & 9 & 13 & & 11 & 12 & & 34 & 3 & 82 \\
\hline$\geq 30$ & & 11 & 15 & & & & & 1 & 34 & 61 \\
\hline Breed & Total & $\begin{array}{l}\text { Japanese } \\
\text { beef cattle }\end{array}$ & 50 & & $\begin{array}{c}\text { Crossbrec } \\
\text { cattle }\end{array}$ & 25 & & $\begin{array}{l}\text { Dairy } \\
\text { cattle }\end{array}$ & 123 & 198 \\
\hline Sex & Total & Bull & 6 & & Steer & 113 & & Cow & 79 & \\
\hline
\end{tabular}

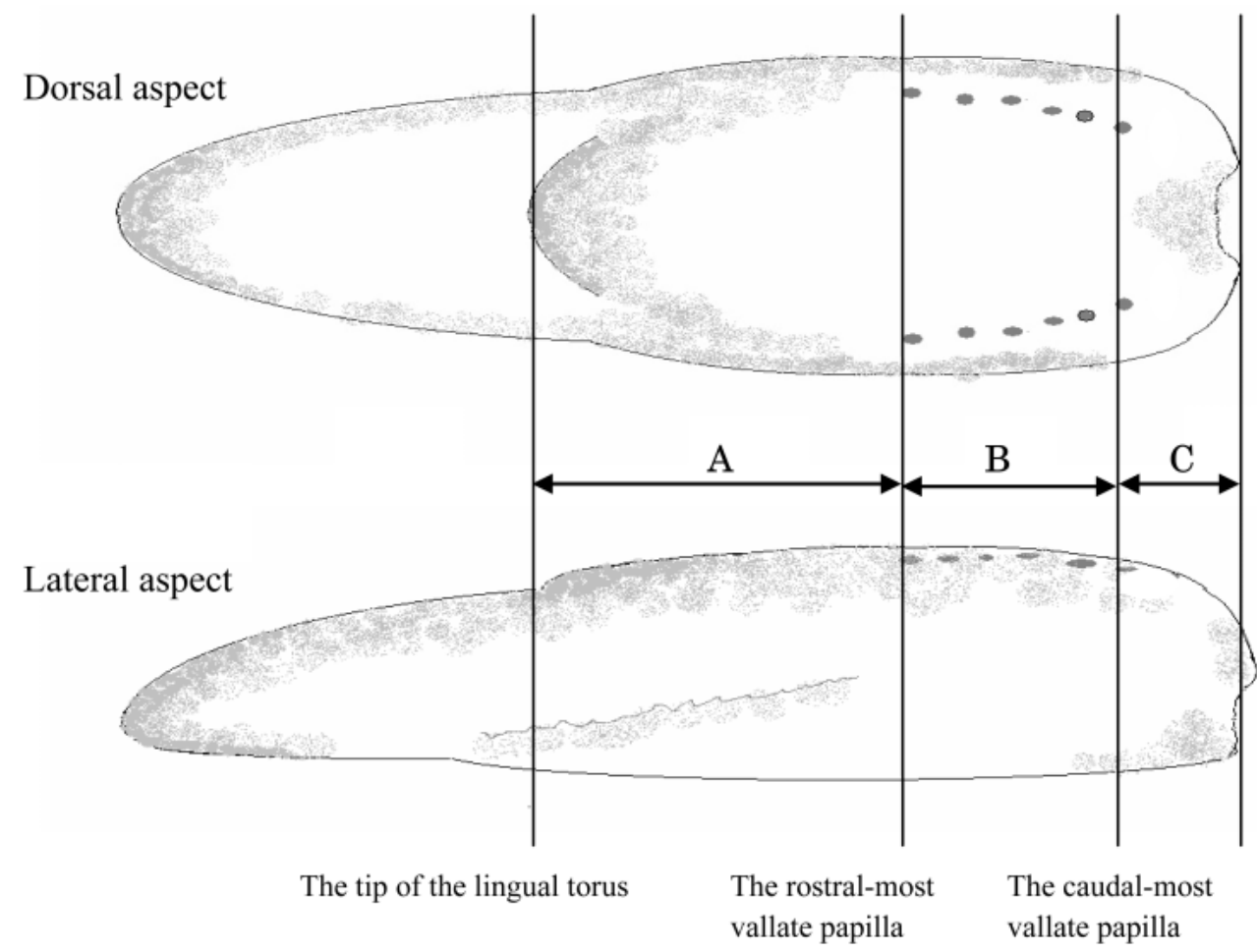

Fig. 1. Areas examined for the distribution of lingual tonsils.

caudal vallate papilla toward the apex linguae (rostrally) or toward the radix linguae (caudally), followed by fixation in $20 \%$ formalin. Then, paraffin sections prepared according to a standard method were stained with hematoxylin-eosin (HE) before microscopic observation of the lamina propria and the muscular layer. To account for individual differences in the specimens, the examination area was divided into three parts: area A (closest to the apex linguae) was defined as the area from the tip of the lingual torus to the most rostral vallate papilla (20 specimens), area B was defined as the area from the most rostral vallate papilla to the most caudal vallate papilla (135 specimens), and area $\mathrm{C}$ was defined as the area from the most caudal vallate papilla to the radix linguae (63 specimens) (Fig. 1). The body of the tongue was divided into dorsal and lateral aspects.
The presence of lingual tonsils was recognized according to histological criteria, specifically, by finding tissues containing crypts and secondary follicles compartmentalized from their surroundings (Fig. 2), and tissues without crypts but with secondary follicles compartmentalized from the surroundings (Fig. 3) [8].

\section{RESULTS}

Detection of lingual tonsils in each area is summarized in Table 2. In all specimens, the distribution of lingual tonsils was limited to the lamina propria and did not extend into the muscular layer. Specimens that had the muscular layer exposed after the removal of the surface mucosa for marketing showed no evidence of lingual tonsillar tissue. 


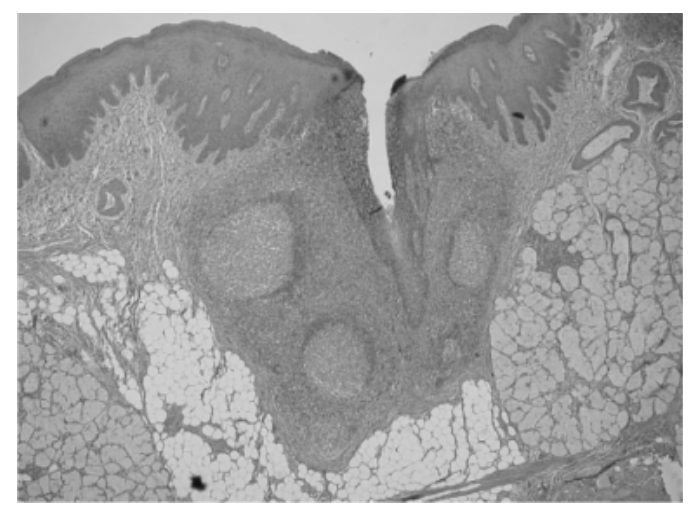

Fig. 2. Lingual tonsils with crypts and secondary follicles.

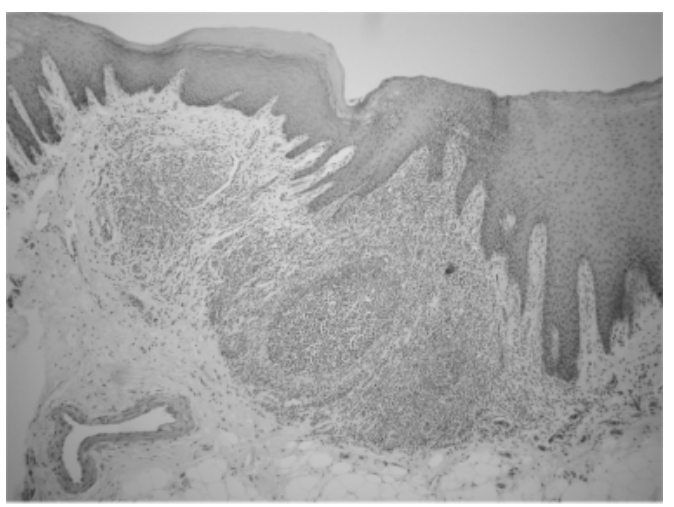

Fig. 3. Lingual tonsils without crypts but with secondary follicles.

The 20 specimens from area A showed no lingual tonsils on the dorsal aspect, but one specimen $(5.0 \%)$ contained lingual tonsillar tissue on the lateral aspect. The lingual tonsillar tissue was located midway between the tip of the lingual torus and the most rostral vallate papilla.

In area $\mathrm{B}$, more than $90 \%$ of the lingual tonsils were located between the radix linguae and the distribution center of the vallate papillae. However, one specimen contained lingual tonsils at the level of the most rostral vallate papilla. In addition, the presence of lingual tonsils was inversely related to the age of specimen. Of the 135 specimens from area B, $39(28.9 \%)$ contained lingual tonsils on the dorsal aspect and $78(57.8 \%)$ contained them on the lateral aspect.

In area $\mathrm{C}$, most of the lingual tonsils were distributed between the most caudal vallate papilla and the radix linguae; specimens from individuals aged less than 30 months always contained tonsils on either the dorsal or lateral aspect, as well as inconsistently in other locations. Of the 63 specimens from area C, $48(76.2 \%)$ contained lingual tonsils on the dorsal aspect and $60(95.2 \%)$ contained them on the lateral aspect.

\section{DISCUSSION}

There are no published reports on the accumulation of prion proteins ( $\mathrm{PrP})$ in the lingual tonsils of BSE-infected cattle. However, the tonsils of sheep have shown infectivity or prion accumulation in the case of natural scrapie [3]. For this reason, EC Regulation 999/2001 specifies that the tongue be cut at the lingual torus of the basihyoid bone to avoid food contamination by lingual tonsils. Wells et al. [11] reported that intracerebral inoculation of palatine tonsils of cattle experimentally infected orally with BSE ten months earlier caused BSE infection in other cattle. They also pointed out the possibility that tonsils in general are infective. Furthermore, they examined the distribution of lingual tonsils in 100 British cattle tongues marketed for consumption and reported that lingual tonsils were still histologically observed in the tongues after removal of the radix linguae. Similarly, Kühne et al. [5] histologically examined the tongues of two cattle aged more than 18 months and reported that lingual tonsils were observed in areas distant from the most caudal vallate papilla.

Our study found that $93.5 \%$ of the lingual tonsils were distributed between the most caudal vallate papilla and the

Table 2. Detection of lingual tonsils in each area

\begin{tabular}{|c|c|c|c|c|c|c|c|c|c|c|}
\hline \multirow[b]{2}{*}{ Area } & & \multicolumn{7}{|c|}{ Age in months } & \multirow{2}{*}{\multicolumn{2}{|c|}{ Total }} \\
\hline & & $<12$ & $<20$ & & $<3$ & & $\geq 30$ & & & \\
\hline \multirow{3}{*}{$\mathrm{A}^{\mathrm{a})}$} & $\mathrm{n}$ & 5 & 5 & & 5 & & 5 & & 20 & \\
\hline & Dorsal aspect & 0 & 0 & & 0 & & 0 & & 0 & \\
\hline & Lateral aspect & 0 & 0 & & 1 & $(20.0)$ & 0 & & 1 & (5.0) \\
\hline \multirow{3}{*}{$\mathrm{B}^{\mathrm{b})}$} & $\mathrm{n}$ & 26 & 25 & & 52 & & 32 & & 135 & \\
\hline & Dorsal aspect & $7 \quad(26.9)$ & 5 & $(20.0)$ & 23 & $(44.2)$ & 4 & $(12.5)$ & 39 & $(28.9)$ \\
\hline & Lateral aspect & $23 \quad(88.5)$ & 18 & $(72.0)$ & 28 & (53.8) & 9 & (28.1) & 78 & (57.8) \\
\hline \multirow{3}{*}{$C^{c)}$} & $\mathrm{n}$ & 0 & 4 & & 30 & & 29 & & 63 & \\
\hline & Dorsal aspect & - & 2 & $(50.0)$ & 25 & $(83.3)$ & 21 & $(72.4)$ & 48 & $(76.2)$ \\
\hline & Lateral aspect & - & 4 & (100.0) & 29 & $(96.7)$ & 27 & $(93.1)$ & 60 & $(95.2)$ \\
\hline
\end{tabular}

a) from the tip of the lingual torus to the most rostral vallate papilla.

b) from the most rostral vallate papilla to the most caudal vallate papilla.

c) from the most caudal vallate papilla to the radix linguae.

The numbers in parentheses indicate detection percentages. 
intermediate position between this vallate papilla and the most rostral vallate papilla. However, in rare cases, lingual tonsils were located near the most rostral vallate papilla or even toward the tip of the lingual torus. Thus, our results reconfirmed past reports.

Moreover examination of lingual tonsils located on the dorsal and lateral aspects of the tongue showed that the tonsils were more commonly found on the lateral aspects of the tongue than on the dorsal aspect, but that their distribution was limited to the lamina propria and did not extend into the muscular layer. This finding was confirmed by the fact that no lingual tonsils were found in the specimens with preexposed muscular layers. Based on our findings that specimens from individuals more than 30 months old had a low prevalence of detection of lingual tonsils, we suggest that lingual tonsils may regress as animals' age.

Based on the above results, we conclude that the removal of the lingual tonsils designated as SRM would be complete if tongues are processed by completely removing the lamina propria layer extending from the tip of the lingual torus to the radix linguae on the dorsal and lateral aspects. The muscular layer can be safely consumed due to its lack of lingual tonsillar tissue.

The study was carried out with the cooperation of 18 meat inspection centers nationwide. The representatives of the centers are as follows: Kazunori Nakanishi (Asahikawa City Meat Hygiene Inspection Center), Hiroya Oyamada (Towada Meat Inspection Center, Aomori Prefecture), Kesayo Saito (Kenpoku Meat Inspection Office, Tochigi Prefecture), Naomi Onuki (Kensei Meat Inspection Office, Ibaraki Prefecture), Susumu Mabara (Kennan Meat Inspection Office, Ibaraki Prefecture), Masakazu Katayama/Kenji Ono (Toso Meat Inspection Station, Chiba Prefecture), Miyuki Hara (Yokohama City Meat Inspection Center), Aki Shimazaki (Nagoya City Meat Inspection Center), Takayoshi Oba (Toyama Prefectural Meat Inspection Center), Yoshinori Kaji (Kanazawa City Meat Inspection Center), Keiko Fujita (Gifu Prefectural Meat Inspection Office), Shoichi Yamanaka (Matsusaka Meat Inspection Office, Mie Prefecture), Tomoyuki Nakayama (Shiga Prefectural Meat Inspection Office), Hanjiro Kitada (Osaka City Meat Inspection Office), Kazuyuki Okahata (Nishiharima Meat Inspection Office, Hyogo Prefecture), Daisuke Nozaki (Kobayashi Meat Inspection Center, Miyazaki Prefecture), Koichi Yamada / Junko Hamada (Sueyoshi Meat Inspection Center, Kagoshima Prefecture), and Tomoyuki Nanba (Shibaura Meat Sanitary Inspection Station, Tokyo Metropolitan Government).
ACKNOWLEDGEMENTS. We thank Dr. Tetsutaro Sata, director of the Department of Pathology, the National Institute of Infectious Diseases and Professor Hidefumi Furuoka of the Department of Veterinary Pathobiological Science, Obihiro University of Agriculture and Veterinary Medicine for their great advice and comments. This study was conducted as a part of "Studies on food-mediated BSE risk Development of slaughter methods for preventing the contamination of meat, etc. by the brain and spinal cord tissues" under the Research on Food Safety (17270701), receiving Health and Labour Sciences Research Grants 2005-2006.

\section{REFERENCES}

1. Dillner, L. 1996. BSE linked to new variant of CJD in humans. BMJ. 30: 312(7034):795.

2. European Commission. 2000. Commission Decision of 29 June $2000,418 / 2000 / \mathrm{EC}$ regulating the use of material presenting risks as regards transmissible spongiform encephalopathies and amending Decision 94/474/EC L158/81.

3. Hadlow, W. J., Kennedy, R. C. and Race, R. E. 1982. Natural infection of Suffolk sheep with scrapie virus. J. Infect. Dis. 146: 657-664.

4. Kato, Y. and Yamauchi, S. 2003. Comparative Anatomical Atlas of Domestic Animals. New edition. Yokendo. Tokyo (in Japanese).

5. Kühne, M., Klein, G. and Gasse, H. 2005. Shortening of the bovine tongue according to regulation (EC) 999/2001 is not complying with the current legal definition of specified risk material - a macroscopical and histological preliminary study, J. Vet. Med. B. 52: 102-104.

6. Law on Special Measures Against Bovine Spongiform Encephalopathy (Law No. 70 of June 14, 2002).

7. SEAC Statement. 2003. Statement on BSE risk from bovine tonsil and consumption of $\mathrm{Ox}$ tongue.

8. The Viscera of the Domestic Mammals. 1979. Verlag Paul Parey, Berlin, Hamburg. pp. 54-55.

9. USDA FSIS Technical service center: Additional Information \& Diagrams Regarding the Removal of Tonsils in Cattle.

10. Wells, G.A., Scott, A.C., Johnson, C.T., Gunning, R.F., Hancock, R.D., Jeffrey, M., Dawson, M. and Bradley, R. 1987. A novel progressive spongiform encephalopathy in cattle. Vet. Rec. 121: 419-420.

11. Wells, G.A., Spiropoulos, J., Hawkins, S.A. and Ryder, S.J. 2005. Pathogenesis of experimental bovine spongiform encephalopathy: preclinical infectivity in tonsil and observations on the distribution of lingual tonsil in slaughtered cattle. Vet. Rec. 156: 401-407.

12. Will, R.G., Ironside, J.W., Zeidler, M., Cousens, S.N., Estibeiro, K., Alperovitch, A., Poser, S., Pocchiari, M., Hofman, A. and Smith, P.G. 1996. A new variant of CreutzfeldtJakob disease in the UK. Lancet 347: 921-925. 